\title{
Dermanyssus gallinae (Acari: Dermanyssidae) a cause of recurrent papular urticaria diagnosed by light and electron microscopy
}

\author{
Original \\ Article \\ Noha S. Ahmed ${ }^{1}$, Asmaa M. El-Kady², Wafaa M. Abd Elmaged ${ }^{3}$, Amal M. \\ Almatary ${ }^{4}$
}

Departments of ${ }^{1,2,4}$ Medical Parasitology and ${ }^{3}$ Dermatology, Faculty of Medicine, ${ }^{1,3}$ Sohag, ${ }^{2}$ South Valley and ${ }^{4}$ Assiut Universities, Egypt

\begin{abstract}
Background: Papular urticaria is a prevalent disturbing condition manifested by chronic or recurring papules caused by a hypersensitivity response to the bites of mosquitoes, bedbugs, fleas, and other insects. D. gallinae was previously indicated as an etiological agent for papular urticaria and chronic itching by several researchers worldwide.

Objective: To throw light on D. gallinae as a cause of papular urticaria in Upper Egypt discussing associating risk factors. Material and Methods: The present study evaluated patients attending the Dermatology Outpatient Clinic, Sohag University Hospitals, Egypt, and who were diagnosed as papular urticaria. Some patients with recurrent papular lesions gave history of exposure to domestic poultry or birds' nests and insect bites. They were selected from 97 patients who complained of itching. They were advised to bring any insects suspected of biting them or that come in contact with their skin, which were then referred to the Parasitology Unit for identification by light (LM) and scanning electron microscopy (SEM).

Results: The examined arthropods were identified as D. gallinae in thirty cases and human fleas in two cases. LM and SEM showed the remarkable identifying features of $D$. gallinae female. Contact with sources of infection (insects) either birds' nests or chicken husbandry was proved to be a risk factor for recurrent papular urticaria. Contact of the same family members to the same source of infection caused prevalence of arthropods borne recurrent papular urticaria among family members. Gender and residence were not associated risk factors.

Conclusion: This is, to the best of our knowledge, the first repot of D. gallinae infestation in Egypt. Pruritic dermatitis caused by D. gallinae (associated with papules and vesicles) may sometimes be mistaken for scabies. Therefore, meticulous history recording, and environmental inspection are necessary to discover the etiology of recurrent papular urticaria.
\end{abstract}

Key Words: Dermanyssus gallinae, dermatitis, pruritus, SEM, Upper Egypt

Received: 6 March 2018, Accepted: 19 June 2018

Corresponding Author: Asmaa M El-Kady, Tel.: 01002240877, E-mail: Asmaa.elkady@med.svu.edu.eg

ISSN: 1687-7942, Vol. 11, No. 2, August 2018.

\section{INTRODUCTION}

Papular urticaria is a prevalent cutaneous lesion and often disturbing manifestation of chronic or recurring papules caused by a hypersensitivity response to insect bites. Individual papules may present as a circle or wheal with a central punctum ${ }^{[1]}$. Children, adult males, non local inhabitants, and those belonging to urban or peri-urban areas may be more vulnerable to papular urticaria ${ }^{[2]}$.

Patients usually record chronic or repeated episodes of a papular burst that tends to take place in groups or clusters associated with intensive pruritus. The burst is distinguished by crops of symmetrically disseminated pruritic papules and papulovesicles ${ }^{[3]}$. The lesions can also be manifested in a region exposed to insect bites and may exist on any body part. The lesions tend to be grouped on bared areas, mostly the extensor surfaces of the extremities.
Sometimes, a centric hemorrhagic punctum may be obvious with ecchymoses and brownish pigmentation persisting after resolution ${ }^{[4]}$.

Fleas, mosquitoes and bedbugs are the insects most commonly associated with these rashes, however any other insects could cause the same skin reaction ${ }^{[5]}$. Fleas and other insects thrive well during summer months and that is why cases of papular urticaria are predominant during these months ${ }^{[1,6]}$. Flea bites produce maculopapular or papular rashes and severe pruritus ${ }^{[7,8]}$. About 50 species of mites can cause lesions in human skin. Cutaneous reaction to mites is usually papular or vesicular, with intense pruritus ${ }^{[9]}$. The chicken mite, Dermanyssus gallinae, is vastly distributed, and was described for the first time in 1778 by de Geer ${ }^{[10]}$. Adults measure around one millimeter in length ${ }^{[11]}$, are colored black, grey or white without host blood in their system; but after feeding mature mites turn red ${ }^{[12]}$. Beside 
adults which ordinarily are found near breeder farms, these mites have four stages in their life cycle: egg, larva, protonymph and deutonymph. D. gallinae mites pose a danger to birds bred for meat and egg production ${ }^{[13]}$. At first it was believed to be a pest of chickens, however, it affects more than 30 species of birds including pigeons, rock doves, sparrows, and starlings. In their absence other available mammals including humans act as hosts ${ }^{[14]}$.

D. gallinae mites cause extreme itching, allergies and dermatitis ${ }^{[12,13]}$. The first human infestation was suspected by Willan in 1809 , and in 1828 de Saint-Vincent was the first to observe the parasite on the skin of an infected individual ${ }^{[15,16]}$. In addition to the ectoparasitic activities, it was declared that D. gallinae mites act as vectors for some bacteria such as Salmonella, Ricketsia, Spirocheta and Pasteurella ${ }^{[17]}$

The objective of our study is to confirm the medical importance of arthropods especially $D$. gallinae as a prevalent causative agent of recurrent papular urticaria in upper Egypt.

\section{MATERIAL AND METHODS}

To achieve our objective, a descriptive analytical study was conducted in the Dermatology Outpatient Clinic, Sohag University Hospitals, in the period from June to September 2017

Selection of patients: Examination of 97 patients who complained of itching revealed erythematous excoriated and some urticated papules in different parts of their body mainly on the extremities. Complete relief of the condition was achieved in 38 patients after medication by steroids and antihistamines. After meticulous recording of history, 32 patients who gave history of insect bites or exposure to domestic poultry or birds' nests, were advised to collect any insects that bite them or come in contact with their skin. These were then referred to the Parasitology Unit, Sohag University, for identification both by light (LM) and scanning electron microscopy (SEM).

Light microscope analysis $(\mathbf{L M})^{[18]}$ : Samples were processed for examination under light microscope. Briefly, samples were passed in $10 \% \mathrm{KOH}$ solution at room temperature for overnight or more according to specimen clearance. Then, samples were passed consecutively in different ethanol gradients $(70 \%, 80 \%, 90 \%, 95 \%$ and finally $100 \%$ ) for dehydration. Following final dehydration, they were doused in xylene before mounting on slides in distyrene (a polystyrene), a plasticiser (tricresyl phosphate), and xylene (DPX). Examination and photographing of the samples were completed in the Parasitology Department, Faculty of Medicine, Sohag University, Egypt.

Scanning electron microscope (SEM) analysis $^{[19]}$ : Arthropods were fixed in 5\% glutaraldehyde for 24 hours followed by: (1) washing in soda-cacodylate acid ( $\mathrm{pH} \mathrm{7.3)} \mathrm{(four} \mathrm{times,} 10 \mathrm{~min} / \mathrm{wash}$ ); (2) fixation in osmium tetraoxide $1 \%$ for 2 hours; (3) washing in sodacacodylate acid (three times, $10 \mathrm{~min} / \mathrm{wash}$ ); and (4) dehydration in a graduated acetone series $(30,50,60,70$, $80,90,95$, and $100 \%, 5$ min each). Samples were then incubated at $20-25^{\circ} \mathrm{C}$, stuck on double scotch tape carbon and coated with gold. Examination and photographs of the samples were performed in SEM Unit, Central Laboratory, Sohag University, Egypt.

Ethical approval and informed consent: Informed consent was obtained from all adult participants and parents of the children included in the study

Statistical analysis: Data analysis were performed using the statistical package for social sciences (SPSS) version 16. Chi-square test was used to assess the significance of association of recurrent papular urticaria with the independent variables. Fischer exact test was used when number of cells is less than 5. $P$ value less than 0.05 was considered statistically significant.

\section{RESULTS}

All 97 patients subjected to the present study, complained of itching and were diagnosed on clinical examination as papular urticaria. The age of participants ranged from 2 to 40 years; $66(68 \%)$ were children up to 10 years, and 31 (32\%) were adults; 65 (67\%) were females; $81(83.5 \%)$ were from urban areas.

On treatment with known antihistaminic and topical steroids $38(39.2 \%)$ patients showed complete recovery with no recurrence of their complaint, while $59(60.8 \%)$ patients suffered recurrence of the condition upon drug suspension (Table 1).

Of the 59 cases with recurrent papular urticaria, 4 $(6.8 \%)$ gave history of chicken husbandry, 26 (44.1\%) recalled the presence of bird nests in the windows of their houses, $2(3.4 \%)$ mentioned exposure to insect bites. The remaining $27(45.7 \%)$ did not mention having contact with any type of insects (Table 2). Ten patients $(16.9 \%)$ were from rural areas, while $49(83.1 \%)$ were from urban areas with no statistical significance $(P>0.05)$. From four families, 27 out of 59 patients $(45.8 \%)$ had the same complaint, while $32(54.2 \%)$ patients did not give history of family involvement $(P<0.05)$. All patients with history of positive family involvement conceded to contact with a source of arthropods, either birds nest (23/27) or chicken husbandry (4/27). On the other hand, among patients with negative history of family involvement, the majority (29/32) of patients gave no history of presence of birds' nests or chicken husbandry and only 3 patients recalled the presence of birds' nests in their houses (Table 2). Therefore, family involvement is not a risk factor for recurrent papular urticaria but exposure of the family members to the same source of infection causes affection of more than one member and predominance of this symptom among families. 
Clinical examination of the 59 patients showed that skin lesions were in the form of papules and crusted lesions. It was observed that $45.8 \%(27 / 59)$ of patients had papular lesions only while $54.2 \%$ (32/59) had both papules and crusted lesions which was statistically non- significant $(P>0.05)$. Skin lesions were apparent on different parts of the body. The most common sites were the exposed areas as legs $(25.4 \%)$ and arms $(27.1 \%)$ in $41(76.3 \%)$ patients; $18(23.7 \%)$ patients had lesions over covered parts of their body with no statistical significance $(P>0.05)$ (Table 3 and Plate I).

Table 1: Patients data and causes of recurrent papular urticaria
Arthropods were identified as D. gallinae in $30(50.8 \%)$ cases and human fleas in $2(3.4 \%)$ cases (Table 1). Using LM, D. gallinae adult female (1 mm long) was identified by its characteristic morphological structures as dorsal shield, long styliform chelicerae in the anterior part and mesostigmatal pore between coxa 3 and coxa 4 (Plate II) SEM examination (Plate III), showed dorsal shield with projecting shoulder that is largely rounded posteriorly, the genitoventral shield broadly rounded posteriorly and the anal shield. Identification of the mite was confirmed by the characteristic lack of setaj 3 on the dorsal shield, reticulation on dorsal shield and ventral shield, and the anal shield with three anal setae around anal opening.

\begin{tabular}{|c|c|c|c|c|c|c|}
\hline \multirow{3}{*}{\multicolumn{2}{|c|}{ Residence/Gender }} & \multirow{2}{*}{\multicolumn{2}{|c|}{$\begin{array}{c}\text { Papular urticaria } \\
\text { Recurrence }\end{array}$}} & \multicolumn{3}{|c|}{ Arthropods involvement } \\
\hline & & & & \multicolumn{2}{|c|}{ Positive } & \multirow{2}{*}{$\begin{array}{l}\text { Negative } \\
\text { No. (\%) }\end{array}$} \\
\hline & & No & Yes & D. gallinae & Flea & \\
\hline \multirow{3}{*}{ Rural } & & & & & & \\
\hline & Female & $5(5.1)$ & $8(8.2)$ & $4(6.7)$ & $1(1.7)$ & $3(5.1)$ \\
\hline & Male & $1(1.1)$ & $2(2.1)$ & $1(1.7)$ & $0(0)$ & $1(1.7)$ \\
\hline \multirow{2}{*}{ Urban } & Female & $21(21.6)$ & $31(31.9)$ & $16(27.1)$ & $1(1.7)$ & $14(23.7)$ \\
\hline & Male & $11(11.3)$ & $18(18.6)$ & $9(15.3)$ & $0(0)$ & $9(15.3)$ \\
\hline \multicolumn{2}{|c|}{ Total } & $38(39.2)$ & $59(60.8)$ & $30(50.8)$ & $2(3.4)$ & $27(45.8)$ \\
\hline
\end{tabular}

Table 2: Risk factors of recurrent papular urticaria

\begin{tabular}{|c|c|c|c|c|c|}
\hline \multirow{2}{*}{\multicolumn{2}{|c|}{$\begin{array}{l}\text { Source } \\
\text { of infection/Gender }\end{array}$}} & \multicolumn{2}{|c|}{ Residence } & \multicolumn{2}{|c|}{ Family involvement } \\
\hline & & Rural & Urban & Negative & Positive \\
\hline \multirow{3}{*}{ Bird nest } & & & & & \\
\hline & Female & $4(0.8)$ & $13(22)$ & $2(3.4)$ & $15(25.4)$ \\
\hline & Male & $1(1.7)$ & $8(13.6)$ & $1(1.7)$ & $8(13.6)$ \\
\hline \multirow{2}{*}{ Chicken husbandry } & Female & $0(0)$ & $3(5.1)$ & $0(0)$ & $3(5.1)$ \\
\hline & Male & $0(0)$ & $1(1.7)$ & $0(0)$ & $1(1.7)$ \\
\hline \multirow{2}{*}{ None } & Female & $4(6.8)$ & $15(25.4)$ & $19(32.1)$ & $0(0)$ \\
\hline & Male & $1(1.7)$ & $9(15.2)$ & $10(17)$ & $0(0)$ \\
\hline \multicolumn{2}{|c|}{ Total } & $10(17)$ & $49(83)$ & $32(54.2)$ & $27(45.8)$ \\
\hline \multicolumn{2}{|c|}{ Statistical analysis } & \multicolumn{2}{|c|}{$\mathrm{P}>0.05$} & \multicolumn{2}{|c|}{$\mathrm{P}<0.05$} \\
\hline \multicolumn{2}{|l|}{ Lesion } & No. & \multirow[t]{2}{*}{$\%$} & \multicolumn{2}{|c|}{ Statistical analysis } \\
\hline \multicolumn{5}{|l|}{ Type } & \\
\hline \multicolumn{2}{|c|}{ Papules and crusts } & \multicolumn{2}{|r|}{54.2} & \multirow{2}{*}{\multicolumn{2}{|c|}{$P>0.05$}} \\
\hline \multicolumn{2}{|c|}{ Papules only } & 27 & 45.8 & & \\
\hline \multicolumn{6}{|l|}{ Site } \\
\hline \multicolumn{2}{|l|}{ Neck } & \multicolumn{2}{|r|}{6.8} & \\
\hline \multicolumn{2}{|l|}{ Chest } & 6 & 10.2 & & \\
\hline \multicolumn{2}{|l|}{ Trunk } & 8 & 13.6 & & \\
\hline \multicolumn{2}{|l|}{ Forearm } & 16 & 27.1 & & 0.05 \\
\hline Hand & & 8 & 13.6 & & \\
\hline Leg & & 15 & 25.4 & & \\
\hline Foot & & 2 & 3.4 & & \\
\hline Total & & 59 & 100 & & \\
\hline
\end{tabular}



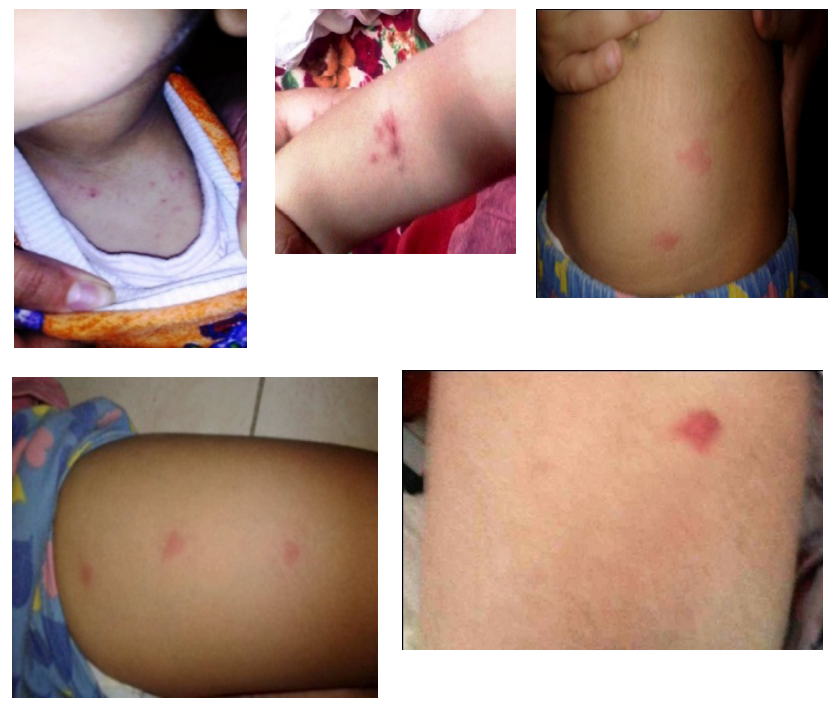

Plate I: Cutaneous manifestations in patients due to infestation by $D$. gallinae. Papular urticaria affecting different parts in the body.
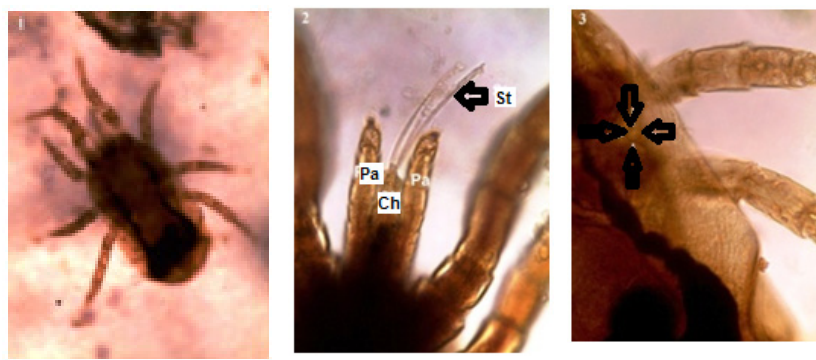

Plate II. Identification of D.gallinae by LM.

Fig. 1: Adult female (1 mm long) with characteristic dorsal shield, Fig. 2: Anterior part with pedipalps $(\mathrm{Pa})$, chelicerae $(\mathrm{Ch})$, with long stylet (St),

Fig. 3: Mesostigmatal pore between coxa 3 and coxa 4 (arrows).
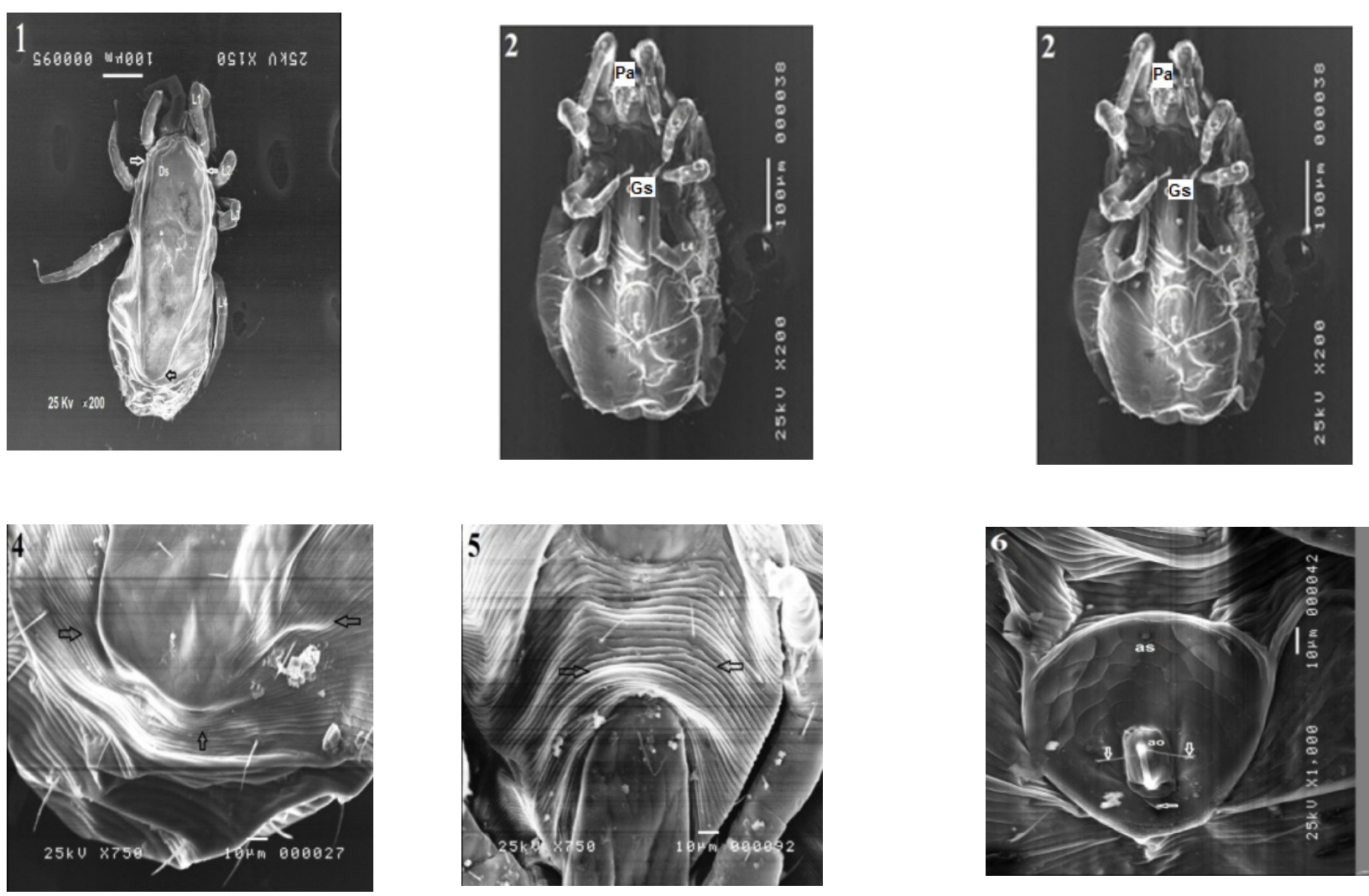

Plate III: Identification of D.gallinae by SEM.

Fig. 1: Dorsal overview showing the idiosoma, Dorsal shield (Ds) with projecting shoulder and largely rounded posteriorly (arrows) and 4 pairs of legs (L1- L4).

Fig. 2: Ventral overview showing pedipalps (Pa), Genito-ventral shield (Gs) broadly rounded posteriorly (arrows) and 4 pairs of legs ( L1L4)

Fig. 3: Lack of seta $\mathrm{j} 3$ on the dorsal shield (arrows).

Fig. 4: Reticulation on dorsal shield (arrows).

Fig. 5: Reticulation on ventral shield (arrows).

Fig. 6: Detail of the anal shield (as) with three anal setae (arrows) around anal opening (ao). 


\section{DISCUSSION}

Papular urticaria is a widespread skin disorder mainly affecting children, manifested by chronic or repetitive papules due to a hypersensitivity stimulus to the bites of mosquitoes, bed bugs, fleas and other insects ${ }^{[20]}$. In the present study, the majority of patients were children and this was consistent with previous studies ${ }^{[2,5,21,22]}$. Cases were recorded in summer months, in agreement with the habits of fleas and other insects that flourish during summer. The majority of patients $(76.3 \%)$ had lesions of papular urticaria over exposed parts of the body which is in accordance with other studies ${ }^{[23,24]}$.

Recurrent papular urticaria in the form of skin rash that lasted for few days and disappeared after medications to reappear in most of our patients was caused by insects. In only a small group of patients, arthropod agents were not involved. Apparently contact with arthropods raised the risk of recurrence of papular urticaria. It is not surprising that many of the patients in this study had history of similar eruptions in their family; all of them gave history of contact with either birds' nests or chicken husbandry, while most of the patients with negative history of family involvement had no history of contact with any source of insects. So occurrence of recurrent papular urticaria in more than one member of the same family is explained by sharing the same environment, exposure to the same source of infection and being bitten by the same arthropods ${ }^{[2]}$. It means that family involvement is not a risk factor for occurrence of recurrent papular urticaria but exposure of family members to the same insect causes prevalence of this disease among families.

Fleas were the causative arthropod in only 2 patients, although fleas have been reported as the most common arthropod cause of papular urticaria ${ }^{[5]}$. However this was an expected result since fleas are difficult to trap.

D. gallinae proved to be the cause of recurrent papular urticaria in the major group of patients. Diagnosis was confirmed both by LM and SEM. D. gallinae was previously detected as an etiology for papular urticaria and chronic itching by several researchers worldwide ${ }^{[15,23,25]}$, but to the best of our knowledge it is the first time to be recorded in Egypt. D. gallinae associated diseases are often linked to synanthropic birds, mostly pigeons and their nests and roosts; multiple cases of nosocomial, army residence, university offices and school infestations have been reported for this mite ${ }^{[26,27]}$.

D. gallinae is a part of the Mesostigmata superfamily. In the absence of its usual host it may attack other mammals including humans causing dermatitis, recognized as papulosquamous rashes and urticarial lesions ${ }^{[28]}$. D. gallinae mites produce pruritus or allergic reactions induced by salivary proteins deposited during feeding ${ }^{[29]}$. The potential medical significance of $D$. gallinae is intensified by the fact that these mites can hold and convey both viral and bacterial zoonotic diseases ${ }^{[30]}$.

Patients in the present study had erythematous papular rashes on different parts of their body including the trunk, arms, front of the neck and thighs. In some cases, erythematous macula-papular rashes were covered with a crust due to violent itching specially on forearms and neck. Rashes were solitary on the trunk in some cases, while in most cases rashes were in the form of few papules on exposed and covered parts of the body. These findings are in accordance with other studies that showed that $D$. gallinae infestations may affect different parts of the body causing intensified itching and pruritic dermatitis usually on the backs of the hands, face, forearms and scalp; and rarely may infest the nares, orbits and eyelids, and genitourinary and rectal orifices ${ }^{[15,31,32]}$.

LM and SEM micrographs identified mites gathered by the patients as $D$. gallinae females which is in accordance with the habits of these mites where mature females must suck blood from their hosts while males occasionally feed on blood ${ }^{[33]}$. Identification of these mites was based on the following characteristic features: idiosoma broadly rounded posteriorly, dorsal shield with eminent shoulder, stylet-like process on the cheliceral article, reticulations of the dorsal and ventral shield, lack of setae $\mathrm{j} 3$ on the dorsal shield, mesostigmatal pores between coxa 3 and 4, and three anal setae around anal opening ${ }^{[29,34]}$. Identification of the cryptic species $D$. gallinae requires both morphological and molecular studies ${ }^{[34]}$.

\section{CONCLUSION}

These cases are of clinical significance because most dermatologists have difficulty identifying ectoparasites that cause new or atypical conditions. This may lead to misdiagnosis with other pruritus and be treated with antihistamines and topical corticosteroids with temporary relief of the symptoms. In such cases, clinical signs and symptoms are usually caused by bites from avian mites that have infested domestic poultry or birds nesting in or near human habitation. Pruritic dermatitis caused by D. gallinae (associated with papules and vesicles) may sometimes be mistaken for scabies, so meticulous history recording, and environmental inspection are necessary to discover and deal with the etiology of papular urticaria.

Author contribution: NS Ahmed and WM Abdel Elmaged conceived the idea. NS Ahmed and AM El-Kady contributed to the interpretation of the results. NS Ahmed, AM El-Kady and AM Almatary participated in the analysis of results and preparation of the manuscript. All authors read and approved the final version of the manuscript. results. 


\section{CONFLICTS OF INTEREST}

We declare that we have no conflicts of interest.

\section{FUNDING}

There was no fund for this work.

\section{REFERENCES}

1. Stibich AS, Schwartz RA. Papular urticaria. Cutis 2001; 68(2): 89-91.

2. Raza N, Lodhi MS, Ahmed S, Dar NR, Ali L. Clinical study of papular urticaria. J Coll Physicians Surg Pak 2008; 18(3): 147-150.

3. Jordaan, H. Francois; Schneider, Johann W. Papular urticaria: A histopathologic study of 30 patients. Am J Dermatopathol 1997; 19(2): 119-126

4. Corazza M, Tassinari M, Pezzi M, Ricci M, Borghi $\mathrm{A}$, Minghetti $\mathrm{S}$ et al. Multidisciplinary approach to Pyemotes ventricosus papular urticaria dermatitis. Acta DermVenereol 2014; 94(2): 248-249.

5. Steen CJ, Carbonaro PA, Schwartz RA. Arthropods in dermatology. J Am Acad Dermatol 2004; 50: 819-842.

6. Howard R, Frieden IJ. Papular urticaria in children. Pediatr Dermatol 1996; 13: 246-249.

7. Chua EC, Goh KJ. A flea-borne outbreak of dermatitis. Ann Acad Med Singapore 1987; 16: 648-650.

8. Chin HC, Ahmad NW, Lim LH, Jeffery J, Hadi AA, Othman $\mathrm{H}$ et al. Infestation with the cat flea, Ctenocephalides felis (Siphonaptera: Pulicidae) among students in Kuala Lumpur, Malaysia. Southeast Asian J Trop Med PublHlth 2010; 41: 1331-1334.

9. Wikel SK: Immune responses to arthropods and their products. Ann Rev Entomology 1982; 27: 21-48

10. Abdigoudarzi M, Mirafzali MS, Belgheiszadeh $\mathrm{H}$. Human infestation with Dermanyssus gallinae (Acari: Dermanyssidae) in a family referred with pruritus and skin lesions. J Arthropod Borne Dis 2013; 8(1): 119123.

11. Roberts V. Diseases of farmyard poultry part 4 External and internal parasites of chickens. NADIS 2013.

12. Hoy MA. Agricultural acarology. Introduction to integrated mite management. CRC Press, Boca Raton, FL 2011; 325-327.
13. Ruff M. Important parasites in poultry production systems. Veter Parasit 1999; 84: 337-347.

14. Sparagano O, George D, Harrington D, Giangaspero A. Significance and control of the poultry red mite, $D$. gallinae. Ann Rev Entomol 2014; 59: 447-466.

15. Akdemir C, Gu“Lcan E, Tanritanir P. Case report: $D$. gallinaein a patient with pruritus and skin lesions. Turkiye Parazitol Derg 2009; 33(3): 242-244.

16. Haag-Wackernagel D, Bircher AJ. Ectoparasites from feral pigeons affecting humans. Dermatol 2010; 220(1): 82-92.

17. Vaiente MC, Chauve C, Zenner L. Experimental infection of Salmonella enteritidis by the poultry red mite, D. gallinae. Vet Parasitol 2007; 146: 329-336.

18. Ottoboni M, Tretola M, Cheli F, Marchis D, Veys P, Baeten $\mathrm{V}$ et al. Light microscopy with differential staining techniques for the characterization and discrimination of insects versus marine arthropods processed animal proteins. Food Additives \& Contaminants: Part A, 2017; 34(8): 1377-1383.

19. Hayat MA (Editor). Principles and technique of electron microscopy. Vol. 1, 2 ed., New Jersey, University Park Press 1981.

20. Naimer SA1, Cohen AD, Mumcuoglu KY, Vardy DA. Household papular urticaria. Isr Med Assoc J 2002; 4: 911- 913.

21. Giraldi S, Ruiz-Maldonado R, Tamayo L, Sosa-deMartinez C. Oral desensitization in papular urticaria in children. Trop Doct 2002; 32: 142-145.

22. Demain JG. Papular urticaria and things that bite in the night. Curr Allergy Asthma Rep 2003; 3: 291-303.

23. Schwartz RA, Gorkiewicz-Petkow A. Bedbug bites eMedicine World Medical Library. Available from http: www.emedicine.com/derm/topic 600.htm. Last update 10.7.2006.

24. Cleary CJ, Buchanan D. Diagnosis and management of bedbugs: an emerging U.S. Infestation. Nurse Pract 2004; $29: 46-48$

25. Bellanger AP, Bories C, Foulet F, Bretagne S, Botterel F. Nosocomial dermatitis caused by D. gallinae. Infect Control Hosp Epidemiol 2008; 29: 282-283.

26. Dogramaci AC, Culha G, Ozçelik S. D. gallinae infestation: an unusual cause of scalp pruritus treated with permethrin shampoo. J Dermatol 2010; 221(5):319-321. 
27. Regan AM, Metersky ML, Craven DE. Nosocomial dermatitis and pruritus caused by pigeon mite infestation. Arch Intern Med 1987; 147:2185- 2187.

28. Fuentes MV, Saintz-Elipe S, Saez-Duran S, GalnPuchades MT. Human ectoparasitism due to the poultry red mite, D. gallinae, in the city of Valencia (Spain) and its surroundings. Rev Iberico-Latinoam Parasitol 2009; 68: 188-191.

29. Pezzi M, Leis M, Chicca M, Roy L. Gamasoidosis by the special lineage L1 of $D$. gallinae (Acarina: Dermanyssidae): a case of heavy infestation in a public place in Italy. Parasitol Intern 2017; 66: 666-670.

30. Scharf MJ, Daly JS. Bites and stings of terrestrial and aquatic life. In: Freedberg IM, Eisen AZ, Wolff K, et al. Eds. Fitzpatrick's dermatology in general medicine. New York: McGraw -Hill 2003; 2261-2298.
31. George DR, Finn RD, Graham KM, Mul MF, Maurer V, Moro CV et al. Should the poultry red mite $D$. gallinae be of wider concern for veterinary and medical science? Parasit Vectors 2015; 8: 178

32. Rossiter A. Occupational otitis external in chicken catchers. J Laryngol Otol 1997; 111: 366-367.

33. Koziatek S, Sokół R. Dermanyssus gallinae still poses a serious threat for the rearing of laying hens. Pol J Natur Sc 2015; 30(4): 451-463.

34. Di Palma A, Giangaspero A, Cafiero MA, Germinara GS. Gallery of the key characters to ease identification of D. gallinae (Acari: Gamasida: Dermanyssidae) and allow differentiation from Ornithonyssus sylviarum (Acari: Gamasida: Macronyssidae). Parasit Vectors 2012; 5: 104 